04

\title{
Газовый электрический разряд с развитой стримерной структурой в квазиоптическом СВЧ пучке
}

\author{
() К.В. Александров, Н.И. Буслеев, Л.П. Грачев, И.И. Есаков, А.А. Раваев \\ Акционерное общество „Московский радиотехнический институт РАН“, \\ 117519 Москва, Россия \\ I e-mail: grachev@mrtiran.ru
}

(Поступило в Редакцию 7 марта 2017 г. В окончательной редакции 27 февраля 2018 г.)

Описаны эксперименты по зажиганию электрического разряда в воздухе атмосферного давления с развитой стримерной структурой. Разряд зажигается в импульсном квазиоптическом линейно поляризованном СВЧ пучке с подкритическим уровнем поля. Пробой воздуха инициируется электромагнитным вибратором, расположенным в свободном пространстве, на поверхности диэлектрической пластины и на внешней или внутренней поверхности кварцевой трубки. По результатам экспериментов оценены скорости распространения фронта разряда навстречу волновому вектору электромагнитной волны.

DOI: 10.21883/JTF.2018.10.46494.2239

\section{Введение}

В работах [1-5] описывается газовый электрический разряд, зажигаемый в квазиоптическом СВЧ пучке с ТЕМ-структурой электромагнитного (ЭМ) поля. Такой разряд при сравнительно высоких газовых давлениях $p$, когда частота столкновений плазменных электронов с молекулами газа $v_{c}$ больше круговой частоты поля $\omega$, реализуется в стримерном виде. При таких давлениях технически, как правило, удается зажечь разряд лишь при уровне исходного поля, меньшем пробойного уровня. В этом случае пробой газа должен быть инициирован. Реализующийся при этом СВЧ разряд в определенном диапазоне величины исходного поля $E_{0}$ и длительности СВЧ импульсов $\tau_{\text {pul }}$ в десятки микросекунд представляет собой развитую систему тонких плазменных каналов.

В настоящей работе описывается разряд данного типа в воздухе. Из экспериментов и теории следует, что плазменные электроны в воздушной разрядной СВЧ плазме имеют энергию в сравнительно узком диапазоне $\varepsilon_{e} \approx 1 \mathrm{eV}$. В этом случае основные коэффициенты, характеризующие ее свойства, можно аппроксимировать сравнительно простыми аналитическими выражениями [6,7]. Так, частота $v_{c} \approx 4 \cdot 10^{9} \cdot p, 1 / \mathrm{s}$, где $p$, как и в аналогичных дальнейших формулах, имеет размерность Ра. Условие электрического пробоя воздуха в пространственно однородном и непрерывном во времени поле определяется зависимостью $v_{i}>v_{a}$, где $v_{a} \approx 2 \cdot 10^{4} \cdot p, 1 / \mathrm{s}-$ частота прилипания плазменных электронов к молекулам воздуха, а $v_{i}=v_{a} \cdot\left(E_{0} / E_{\mathrm{cr}}\right)^{5.34}, 1 / \mathrm{s}$ - частота ионизации молекул воздуха электронными ударами. В этой формуле $E_{0}-$ амплитуда электрической составляющей поля ЭМ волны, а при $v_{c}>\omega$ поле $E_{\mathrm{cr}}=42 \cdot p, \mathrm{~V} / \mathrm{cm}-$ амплитуда электрической составляющей ЭМ поля критического уровня, которая обеспечивает равенство $v_{i}=v_{a}$.

В работе [5] описан способ инициации электрического пробоя воздуха при подкритическом уровне исходного поля $E_{0}<E_{\mathrm{cr}}$ в линейно поляризованном $\mathrm{CBЧ} \mathrm{пучке.}$
Он подразумевает помещение в пучок вдоль вектора $\mathbf{E}_{0}$ ЭМ вибратора. Вибратор представляет собой тонкий металлический пруток длиной $2 L \approx \lambda / 2$, где $\lambda$ - длина волны ЭМ поля. В этой же работе приводятся данные, что при $\lambda=8.9 \mathrm{~cm}$, при атмосферном $p$ воздуха, уровне $E_{0} \geq(1.5-2) \mathrm{kV} / \mathrm{cm}$ и $\tau_{\text {pul }}=40 \mu \mathrm{s}$ в ЭМ пучке в свободном пространстве реализуется подкритический инициированный СВЧ разряд с пространственно развитой стримерной структурой. При расположении вибратора на поверхности диэлектрической пластины, которая помещена в СВЧ-пучок вдоль его оси в $\mathbf{E}_{0}-\mathbf{k}$ плоскость, где $\mathbf{k}-$ волновой вектор ЭМ пучка, при этих же условиях разряд также имеет развитую стримерную структуру, но на поверхности пластины.

В настоящей работе описываются эксперименты по зажиганию такого вида разряда не только в свободном пространстве и на поверхности диэлектрической пластины, но и на внешней или внутренней поверхности кварцевой трубки.

\section{Экспериментальные условия}

Эксперименты выполнялись на описанной в работе [5] установке с $\lambda=8.9 \mathrm{~cm}$ при $\tau_{\text {pul }}=40 \mu \mathrm{s}$. В этой установке со стороны радиопрозрачной торцевой плоскости герметичной цилиндрической рабочей камеры вдоль ее оси может излучаться линейно поляризованная ЭМ волна с ТЕМ-структурой поля. Характерный поперечный размер излучаемого в камеру ЭМ пучка равен $60 \mathrm{~cm}$. Волна отражается от поверхности сферически вогнутого металлического зеркала, которое находится у противоположного торца рабочей камеры и фокусируется в ее центральной области. Расстояние геометрической „точки“ фокуса от среза зеркала $x_{f} \approx 10.5 \mathrm{~cm}$. При этом эксперименты показали, что амплитуда электрической составляющей ЭМ волны $E_{0}$ в фокусной области вдоль оси ЭМ пучка с точностью $80 \%$ остается неизменной в диапазоне $x$ от 4.5 до $12 \mathrm{~cm}$. Распределение $E_{0}$ в этой 
области вдоль вектора $\mathbf{E}_{0}$ имеет вид $E_{0}=E_{0 \max } e^{-\left(y / y_{0}\right)^{2}}$, где начало координат оси $y$ совпадает с осью ЭМ пучка, a $y_{0}=5.2 \mathrm{~cm}$ и в поперечном $\mathbf{E}_{0}$ направлении по оси $z$, оно имеет аналогичный вид с $\mathbf{E}_{0}=E_{0 \max } e^{-\left(z / z_{0}\right)^{2}} \mathrm{c}$ $z_{0}=2.5 \mathrm{~cm}$.

При проведении описываемых опытов величина поля $E_{0 \max }$ была уточнена экспериментально. В них на оси ЭМ пучка при $x=x_{f}$ помещался металлический шарик диаметром $2 a=5 \mathrm{~mm}$. Шарик по оси имел отверстие диаметром $1 \mathrm{~mm}$ и подвешивался на пропущенной через это отверстие капроновой нити, перпендикулярной вектору $\mathbf{E}_{0}$. Заполненная атмосферным воздухом рабочая камера установки может откачиваться. В опытах с точностью $3 \cdot 10^{2} \mathrm{~Pa}$ было определено максимальное давление воздуха $p_{\text {br }}=2.85 \cdot 10^{4} \mathrm{~Pa}$, при котором шарик начинал инициировать СВЧ пробой. При этом для обеспечения наличия начальных электронов в приполярных пробойных областях шарика с $E>E_{\mathrm{cr}}$ в течение СВЧ импульса он облучался ультрафиолетовым (УФ) излучением. Это значение $p_{\text {br }}$ по изложенной в работе [5] методике дало значение $E_{0 \max }=4.4 \mathrm{kV} / \mathrm{cm}$. Следовательно, описываемые эксперименты выполнялись при мощности СВЧ пучка

$$
P_{\text {beam }}=\left(E_{0 \max }^{2} / 2 Z_{0}\right) \cdot\left(\pi y_{0} z_{0} / 2\right)=530 \mathrm{~kW},
$$

где $Z_{0}=120 \pi, \Omega$ - волновое сопротивление свободного пространства.

\section{Результаты экспериментов}

Во всех основных опытах давление воздуха в рабочей камере было атмосферным $p=76 \cdot 10^{3} \mathrm{~Pa}$. СВЧ пробой воздуха инициировался ЭМ вибратором, выполняемым из медного провода диаметром $2 a=0.3 \mathrm{~mm}$. Конкретные длины вибраторов $2 L$ будут указаны в процессе описания экспериментов. В опытах расстояние от вибратора до среза фокусирующего зеркала выдерживалось одинаковым $x_{\text {vib }}=15 \mathrm{~cm}$. Оцененная по изложенной выше методике при этом размере $x_{\text {vib }}$ локальная величина поля на оси пучка $E_{0 \max }=3 \mathrm{kV} / \mathrm{cm}$.

На рис. 1 приведены фотографии разряда при расположении ЭМ вибратора в свободном пространстве. Так как в каждом СВЧ импульсе конкретный вид разрядов несколько отличался, то на рис. 1 приведены его реализации в трех СВЧ импульсах. На фотографиях, как и на всех аналогичных, фокусирующее зеркало расположено слева, т.е. отраженная от него ЭМ волна распространяется слева направо. Ее вектор $\mathbf{E}_{0}$ вертикален. Инициатор параллелен $\mathbf{E}_{0}$ и на фотографиях располагается примерно на правой границе разрядной области. Его длина $2 L=45 \mathrm{~mm}$ и может служить масштабом изображений. На концах инициатора находятся сравнительно яркие плазменные „точки“. Вибратор расположен симметрично оси ЭМ пучка. В своем центре он укреплен на перпендикулярной капроновой нити. „Точка“ его крепления также светится. Светится и капроновая нить.

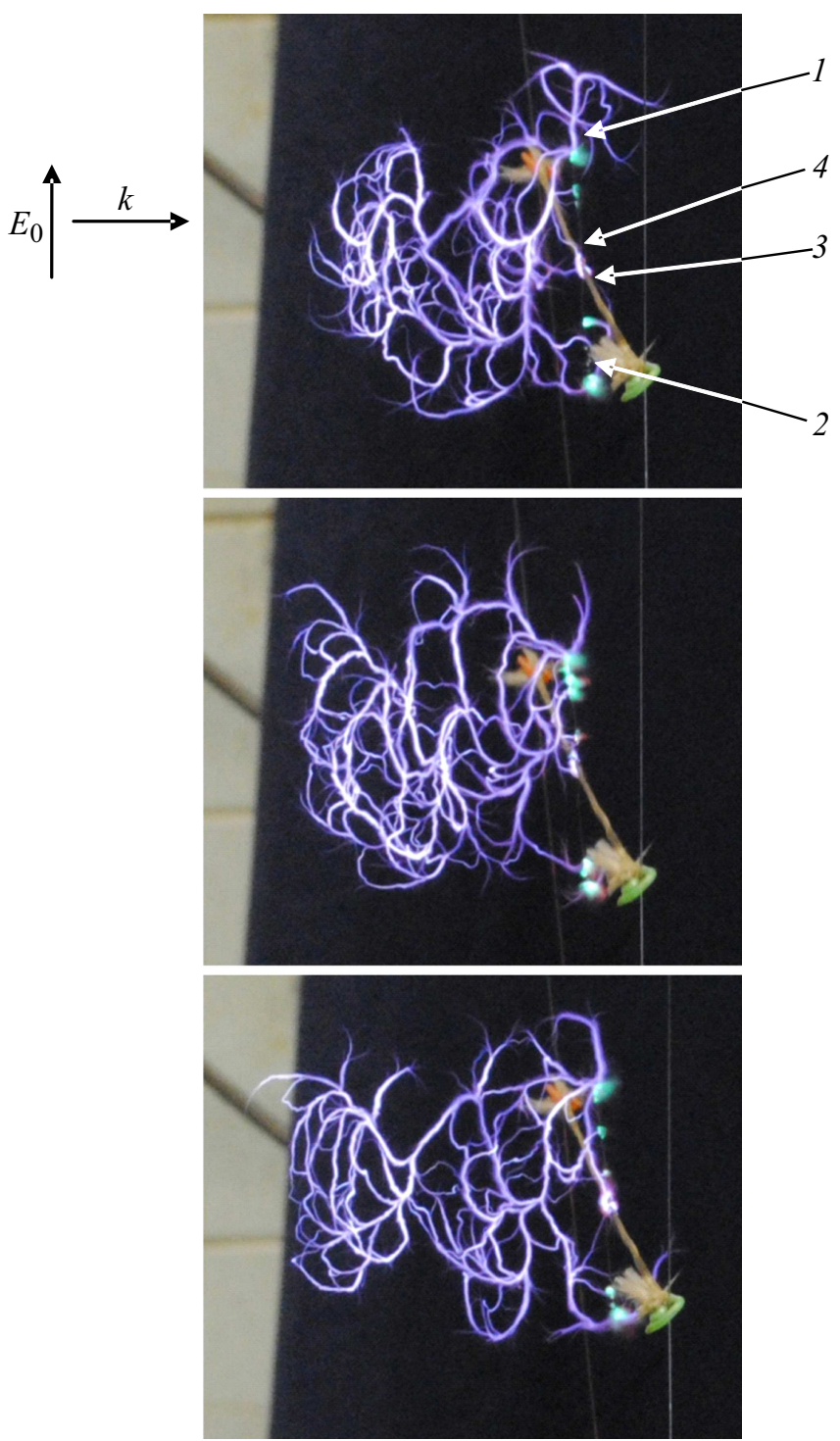

Рис. 1. Инициированный ЭМ вибратором стримерный СВЧ разряд в свободном пространстве: 1 - верхний конец вибратора, 2 - нижний конец вибратора, 3 - капроновая нить, 4 - „точка“ккепления вибратора.

Из фотографий следует, что разрядная область представляет собой объемную систему плазменных каналов. По изображениям может быть оценен средний по импульсам размер этой области навстречу ЭМ излучению $x_{\text {dis } 1}=9 \mathrm{~cm}$.

В работе [5] показано, что этот разряд является динамическим плазменным объектом. „Физику“ его формирования иллюстрирует представленная на рис. 2 заимствованная из этой работы фотография этого вида разряда на раннем временном этапе его развития. На ней справа находится инициирующий пробой ЭМ вибратор длиной $2 L=40 \mathrm{~mm}$.

Представленное на рис. 2 изображение может быть прокомментировано следующим образом. С момента включения СВЧ импульса первоначально на концах- 


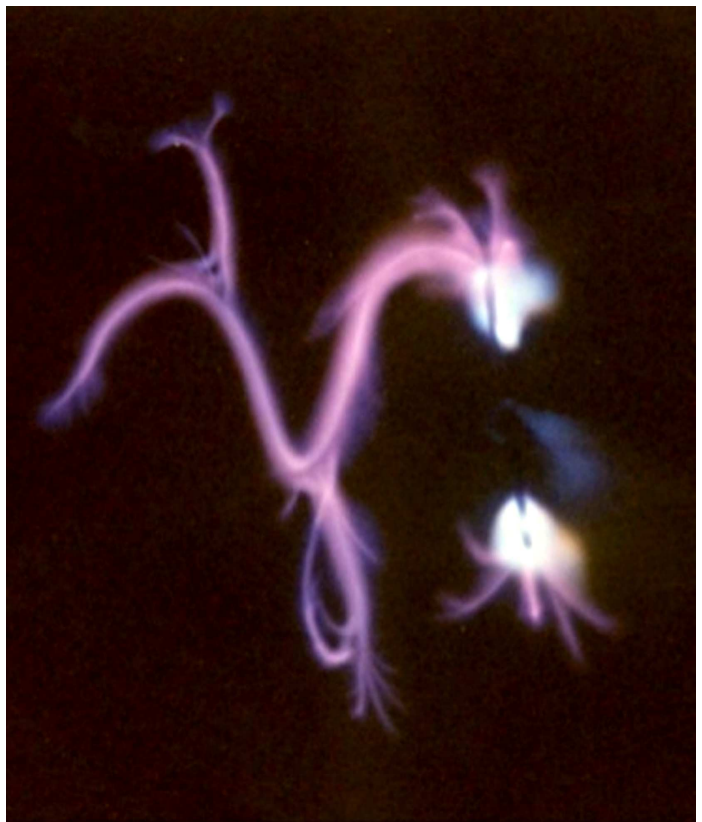

Рис. 2. Динамика развития инициированного ЭМ вибратором стримерного СВЧ разряда.

полюсах вибратора, где индуцированное вибратором поле $E_{\text {ind }}$ на порядки превышает исходное поле $E_{0}$ и больше $E_{\mathrm{cr}}$, развиваются разрядные диффузные плазменные образования. В них вдоль силовых линий $E_{\text {ind }}$ развиваются сравнительно короткие ионизационно-перегретые (ИП) каналы. В процессе развития разряда один или несколько из этих каналов начинают удлиняться. На рис. 2 у верхнего полюса инициатора эту способность приобрел лишь один канал. Удлиняющийся плазменный канал-стример растет по силовой линии индуцированного поля исходного вибратора и образует уже плазменный ЭМ вибратор. Как и положено, когда его длина $2 L$ начинает превышать резонансную длину, индуцированное поле на конце этого плазменного вибратора $E_{\text {pol }}$ уменьшается, и при $E_{\text {pol }} \approx E_{\text {cr }}$ рост СВЧ стримера прекращается. На конце плазменного вибратора вновь развивается диффузное плазменное образование. В нем формируются ИП каналы. Один или несколько из них начинают удлиняться и т.д.

В течение СВЧ импульса эта разрядная система постепенно заполняет поперечное сечение ЭМ пучка, распространяясь преимущественно навстречу вектору $\mathbf{k}$ ЭМ излучения. Оцененная по рис. 1 средняя по импульсам скорость фронта ее распространения $v_{\mathrm{fr} 1}=x_{\text {dis } 1} / \tau_{\text {pul }} \approx 2 \cdot 10^{3} \mathrm{~m} / \mathrm{s}$.

В следующих опытах в ЭМ пучок вдоль его оси и симметрично ей в плоскости $\mathbf{E}_{0}-\mathbf{k}$ пучка помещалась стеклотекстолитовая пластина толщиной $1 \mathrm{~mm}$ и шириной $56 \mathrm{~cm}$. Ее прилегающий к зеркалу край повторял его внутреннюю дугообразную конфигурацию. Противоположный ровный по оси $у$ край пластины имел координату $x=21 \mathrm{~cm}$.
На рис. 3 приведены фотографии трех вариантов реализующегося в этих условиях СВЧ разряда. В опытах пробой воздуха инициировался помещенным на поверхности пластины ЭМ вибратором длиной $2 L=16 \mathrm{~mm}$. На фотографиях он расположен с правой стороны изображений, и у его концов находятся относительно яркие разрядные „точки“.
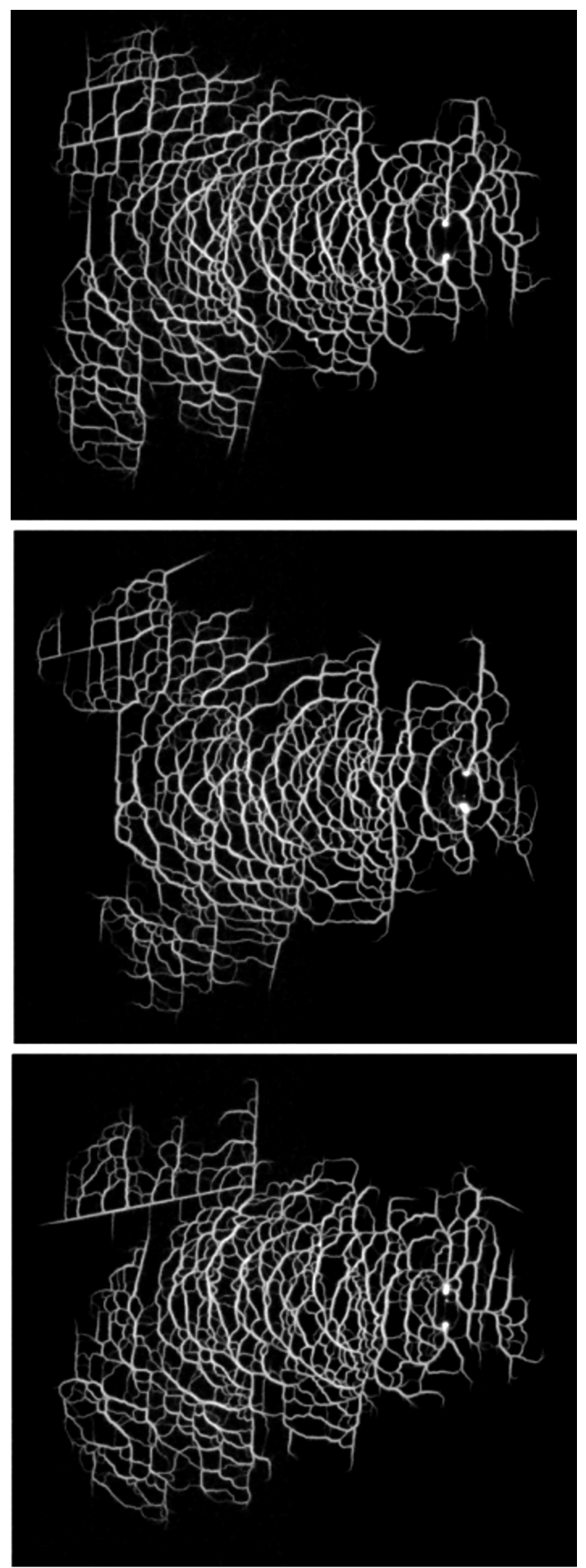

Рис. 3. Инициированный ЭМ вибратором стримерный СВЧ разряд на поверхности диэлектрической пластины. 

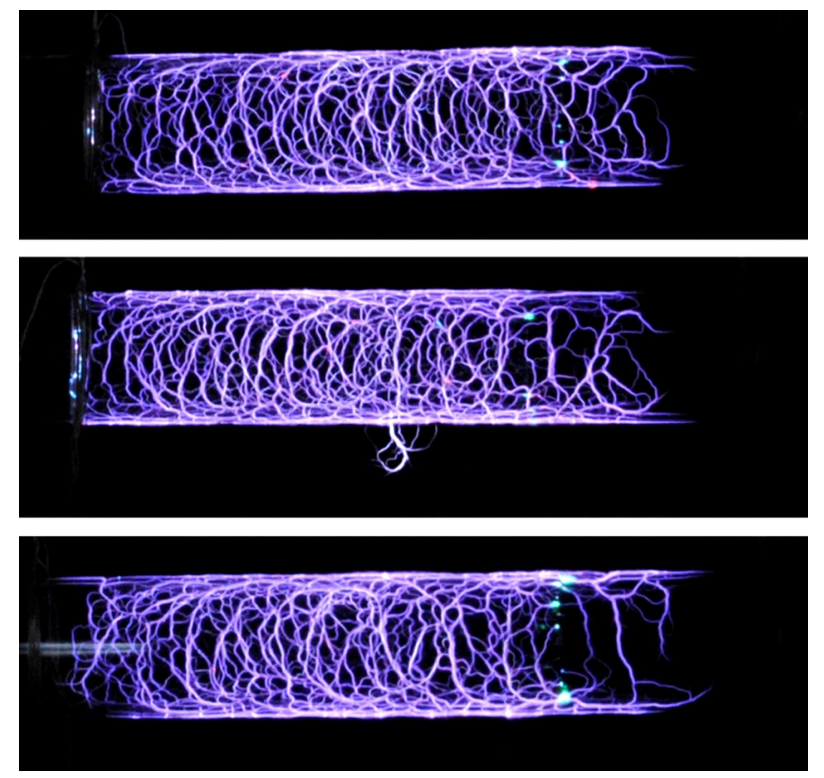

Рис. 4. Инициированный ЭМ вибратором стримерный СВЧ разряд на наружной поверхности кварцевой трубки.
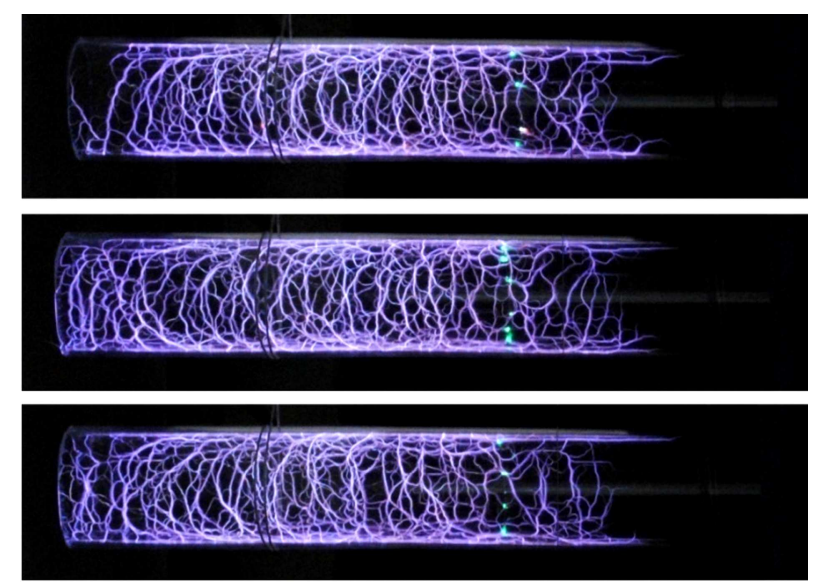

Рис. 5. Инициированный ЭМ вибратором стримерный СВЧ разряд на внутренней поверхности кварцевой трубки.

Фотографии иллюстрируют, что и в этом случае СВЧ разряд представляет собой развитую систему плазменных каналов, но прилегающих к поверхности диэлектрической пластины.

Исследования динамики развития этого вида разряда показали, что она в определенном смысле повторяет описанную выше динамику разряда, реализующегося в свободном пространстве. Эта динамика подразумевает наличие плазменных „полуволновых“ ЭМ вибраторов. Вдоль них гармонически распределяется индуцированный и переменный во времени с частотой $f=\omega / 2 \pi$ „избыточный“ заряд [5]. В этом случае теоретически под поверхностью диэлектрика как бы находятся аналогичные заряды, но противоположного знака. В соответствии с законом Кулона они притягивают реальные „избыточные“ заряды. Так, если над диэлектрической поверхностью на расстоянии $h$ находится заряд $q$, то на него в сторону диэлектрической поверхности действует сила

$$
F=\left(1 / 4 \pi \varepsilon_{0}\right) \cdot\left(q^{2} /(2 h)^{2}\right)(\varepsilon-1) /(\varepsilon+1), \mathrm{N},
$$

где $\varepsilon$ - диэлектрическая проницаемость материала пластины и $\varepsilon_{0}=8.85 \cdot 10^{-12}, \mathrm{~F} / \mathrm{m}$ - диэлектрическая проницаемость вакуума. Отметим, что у стеклотекстолита используемой в опытах пластины $\varepsilon=7$ [8]. Определение масштабов величины $q$ и размера $h$ в конкретных случаях требует дальнейших экспериментальных и теоретических исследований.

Оцененный по приведенным на рис. 3 фотографиям средний размер по $\mathbf{k}$ разрядной области $x_{\mathrm{dis} 2}=16 \mathrm{~cm}$. Его отношение к размеру $x_{\text {dis } 1}$ примерно равно 1.67 , что подтверждает идентичность динамики развития этого вида разряда как в свободном пространстве, так и по поверхности диэлектрика. Действительно, в первом случае плазменные каналы имеют возможность распространяться в пяти направлениях: вперед, вверх, вниз, налево и направо; а во втором - только в трех: вперед, вверх и вниз. Отношение 5/3 также примерно равно 1.67. Размер $x_{\text {dis } 2}$ соответствует скорости $v_{\text {fr } 2}=x_{\text {dis } 2} / \tau_{\text {pul }}=4 \cdot 10^{3} \mathrm{~m} / \mathrm{s}$.

В следующей экспериментальной серии вдоль оси ЭМ пучка в районе фокусной области помещалась кварцевая трубка с внутренним диаметром $36 \mathrm{~mm}$, наружным - $40 \mathrm{~mm}$ и длиной $225 \mathrm{~mm}$. По ее наружной поверхности при $x_{\mathrm{vib}}=15 \mathrm{~cm}$ в перпендикулярной $\mathbf{E}_{0}-\mathbf{k}$ плоскости „примерно“ вдоль $\mathbf{E}_{0}$ был укреплен инициирующий пробой воздуха ЭМ вибратор с $2 L=45 \mathrm{~mm}$.

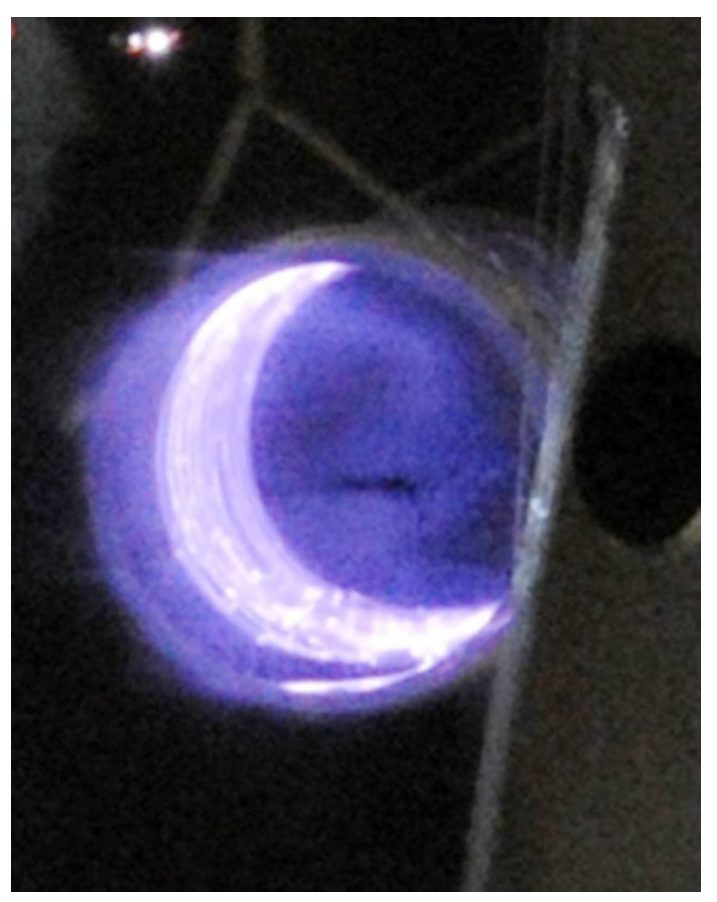

Рис. 6. Торцевой вид инициированного ЭМ вибратором стримерного СВЧ разряда на внутренней поверхности кварцевой трубки. 
Соответствующие фотографии развивающихся при этом разрядов в трех СВЧ импульсах приведены на рис. 4. На них по-прежнему вибратор расположен в правой области изображений и его концевые „точки“ светятся. Масштабом изображений может служить наружный диаметр трубки.

Из фотографий следует, что и в этом случае разряд имеет поверхностно развитую стримерную структуру, т.е. в основном распространяется по наружной поверхности кварцевой трубки. Средняя фотография иллюстрирует, что в некоторых СВЧ импульсах стримерные каналы разряда в фокусной области ЭМ пучка могут отрываться от поверхности трубки.

В опытах средний размер разрядной области от инициатора до ее фронта $x_{\mathrm{dis} 3}=14 \mathrm{~cm}$. Следовательно, при реализации разряда по внешней поверхности трубки $v_{\mathrm{fr} 3}=3.5 \cdot 10^{3} \mathrm{~m} / \mathrm{s}$. Эта величина близка к значению $v_{\mathrm{fr} 2}$.

В заключительной экспериментальной серии на этой же трубке инициирующий пробой воздуха вибратор длиной $2 L=45 \mathrm{~mm}$ при $x_{\mathrm{vib}}=15 \mathrm{~cm}$ был укреплен уже на внутренней ее поверхности.

И в этом случае разряд представлял собой систему плазменных каналов, распространяющихся навстречу вектору $\mathbf{k}$ ЭМ пучка по внутренней поверхности диэлектрической трубки. Соответствующие фотографии приведены на рис. 5. Они иллюстрируют, что размер $x_{\text {dis } 4}$ в различных СВЧ импульсах имеет статистический разброс. Так, из двух нижних фотографий следует, что

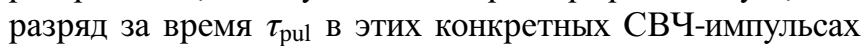
распространился уже до левого среза трубки и мог бы распространиться и несколько дальше. Таким образом, реализованный разряд при конкретной геометрии его реализации имеет $x_{\text {dis } 4} \gtrsim 15 \mathrm{~cm}$ и скорость распространения этого вида разряда $v_{\mathrm{fr} 4} \gtrsim 3.7 \cdot 10^{3} \mathrm{~m} / \mathrm{s}$.

То, что разряд этого вида распространяется именно по внутренней поверхности кварцевой трубки, не отрываясь от нее, иллюстрирует фотография, приведенная на рис. 6. Для регистрации этого изображения у левого среза трубки под углом в $45^{\circ}$ к ее оси помещалось зеркало и фиксировалось изображение разряда на нем.

\section{Заключение}

Таким образом, экспериментально реализован инициированный ЭМ вибратором СВЧ разряд в воздухе при атмосферном давлении в линейно поляризованном квазиоптическом СВЧ пучке с подкритическим уровнем исходного поля. Разряд зажжен в свободном пространстве, на плоской диэлектрической поверхности, на наружной поверхности диэлектрической трубки и на ее внутренней поверхности. Во всех случаях разряд представляет собой развитую систему тонких плазменных каналов, распространяющихся преимущественно навстречу ЭМ волне. В опытах скорость распространения разрядного фронта имеет масштаб нескольких единиц, умноженных на $10^{3} \mathrm{~m} / \mathrm{s}$.
В настоящее время у разряда этого вида, зажигаемого в свободном пространстве, физически ясны все этапы его развития. Количественно же могут быть оценены диффузный и ИП этапы [5]. Есть вопросы к стримерному этапу. Это прежде всего касается скорости роста СВЧ стримеров и параметров образующихся при этом плазменных каналов. В тоже время особый физикотеоретический интерес представляет этот вид разряда, зажигаемый на диэлектрической поверхности. Это влияние на его динамические характеристики свойств диэлектрика, т. е. величины его диэлектрической проницаемости $\varepsilon$ и тангенса угла диэлектрических потерь $\operatorname{tg} \delta$. Параметры диэлектрика могут повлиять и на тепловые характеристики различных участков разряда. В случае его зажигания на поверхности диэлектрической трубки требует выяснения и зависимость свойств разряда от диаметра трубки.

Одно из направлений практического применения данного вида разряда может быть связано с его использованием при зажигании на внутренней поверхности диэлектрической трубки. Если эту трубку заполнить газовой горючей смесью, то такой вид разряда может осуществить практически „мгновенный“ многоочаговый ее поджиг вдоль трубки. При этом оценки показывают, что для реализации энергетически выгодного детонационного режима горения [9] суммарная энергия поджига существенно сократится по сравнению с энергией традиционного способа детонационного поджига лишь в одном поперечном сечении канала.

\section{Список литературы}

[1] Батанов Г.М., Грицинин С.И., Коссый И.А., Магунов А.Н., Силаков В.П., Тарасова Н.М. // Тр. Физического института им. П.Н. Лебедева АН СССР. 1985. Т. 160. С. 174-203.

[2] Высокочастотный разряд в волновых полях // Сб. науч. тр. ИПФ АН СССР / Отв. ред. А.Г. Литвак. Горький, 1988.

[3] Радиофизика. Сборник научных трудов МРТИ АН ССР / Гл. ред. Г.И. Бацких. М.: МРТИ, 1991.

[4] Зарин А.С., Кузовников А.А., Шибков В.М. Свободно локализованный СВЧ разряд в воздухе. М.: Нефть и газ, 1996.

[5] Грачев Л.П., Есаков И.И., Александров К.В., Раваев А.А., Северинов Л.Г., Ходатаев К.В. Газовый электрический разряд в квазиоптическом СВЧ пучке. М.: АО „МРТИ PAH“, 2015. $187 \mathrm{c}$.

[6] Борисов Н.Д., Гуревич А.В., Милих Г.М. Искусственная ионизированная область в атмосфере. М., 1986. 184 с.

[7] Лупан Ю.А. // ЖТФ. 1976. Т. 46. Вып. 11. С. 2321-2326.

[8] Курлин М.В., Панова Я.И., Пасынков В.В., Таиров В.Н. Электрорадиоматериалы. Л.: Судостроение, 1969. 343 с.

[9] Зельдович Я.Б. // ЖТФ. 1940. № 1(17). С. 1453-1461. 